\title{
INSTITUCIONALIZACIÓN Y PROFESIONALIZACIÓN DE LA SOCIOLOGÍA ARGENTINA: REVISANDO LA TRAYECTORIA DE JOSÉ ENRIQUE MIGUENS ${ }^{1}$
}

\author{
Leandro Aramburu * \\ Guido Giorgi ** \\ Universidad de Buenos Aires, Argentina
}

http://dx.doi.org/10.5209/rev_NOMA.2013.42340

\begin{abstract}
Resumen.- La historiografía de la sociología argentina ha tenido como personaje principal a Gino Germani, y como escenario privilegiado, a la Universidad de Buenos Aires. Sin embargo, el proceso de constitución de un campo relativamente autónomo de la sociología comprende también actores, espacios de socialización e instituciones que han quedado ausentes de los principales relatos historiográficos de esta disciplina. Por esta razón, la reconstrucción de trayectorias alternativas a la de Germani nos permitirá profundizar el conocimiento sobre el proceso de institucionalización y profesionalización de esta disciplina en la Argentina. Este interrogante nos lleva directamente a revisar la trayectoria del Dr. José Enrique Miguens. Abogado de profesión, se especializó en Sociología Económica con Parsons y Sorokin en la Universidad de Harvard (EEUU), entre 1944 y 1945. Fue docente de sociología en la UBA durante los primeros gobiernos peronistas, fundador de la segunda carrera de sociología del país, e impulsor de la consultoría privada a partidos políticos y empresas. Efectivamente, Miguens se revela como un actor central de la sociología argentina. Sin embargo, se trata de un capítulo aún no escrito en la historia de esta disciplina. El objetivo de este artículo es realizar un recorrido por la trayectoria tanto académica, intelectual, política y profesional de Miguens. Esto nos permitirá comprender procesos más amplios, que tienen que ver con la historia de la sociología argentina y su interrelación con procesos políticos, culturales y sociales, que se hacen carne en las acciones, decisiones y representaciones de cada individuo.
\end{abstract}

Palabras clave.- institucionalización, profesionalización, Sociología Argentina, intelectuales católicos, José Enrique Miguens

\footnotetext{
${ }^{1}$ Este artículo es la reformulación de una ponencia presentada en el "Primer Congreso de Sociólogos de la Provincia de Buenos Aires" (Julio 2009). La misma fue producto de una investigación realizada en el marco del Proyecto UBACyT S425 (2008-2010), "Institucionalización y profesionalización de la sociología en Argentina (1940-2000)", bajo la dirección del Dr. Diego Pereyra.

* Sociólogo (UBA). Becario por el Gobierno de Aragón (España), para realizar la Maestría en Sociología de las Políticas Públicas y Sociales, Universidad de Zaragoza (UNIZAR). Investigador del Grupo de Investigación en Historia de la Sociología Argentina, con sede en el Instituto de Investigaciones Gino Germani (IIGG-UBA), bajo la dirección del Dr. Diego Pereyra. E-mail: leandroaramburu@gmail.com

${ }^{* *}$ Sociólogo (UBA). Becario doctoral CONICET, programa Sociedad, Cultura y Religión, CEIL. Docente Facultad de Ciencias Sociales y Facultad de Filosofía y Letras, UBA. Investigador del Grupo de Investigación en Historia de la Sociología Argentina, con sede en el Instituto de Investigaciones Gino Germani (IIGG-UBA), bajo la dirección del Dr. Diego Pereyra. E-mail: giorgiguido@gmail.com
} 


\title{
Institutionalization and professionalization of Argentinean Sociology: Reviewing the career of José Enrique Miguens
}

\begin{abstract}
The historiography of Argentine sociology has had Gino Germani as the main character, and the University of Buenos Aires as the privileged scene. However, the formation process of a relatively independent field of sociology also include actors, socializing spaces and institutions that remain away from the main historiographical accounts of this discipline. For that reason, the reconstruction of alternative paths will increase the knowledge about the process of institutionalization and professionalization of that discipline in Argentina. This question leads directly to review the career of Dr. José Enrique Miguens. After becoming a lawyer, he specialized in economic sociology with Parsons and Sorokin in Harvard University (USA), between 1944 and 1945. Then he taught sociology at the University of Buenos Aires during the Peron's governments (1946-1955). After that, he found the second degree in sociology of Argentina, and at the same time he promoted private consulting for political parties and companies. Indeed, Miguens has shown himself to be a main character in Argentine sociology. However, it remains an unwritten chapter in the history of this discipline. The aim of this article is to make an overview of the different areas in Miguens career, such as academic, intellectual, professional and politic. This will allow understanding institutionalization and professionalization processes of Argentine sociology and its relationship with political, cultural and social spheres of the Argentine society, where individuals with its actions, decisions and performances play a key role.
\end{abstract}

Keywords.- institutionalization, professionalization, Argentina Sociology, catholic intellectuals, Jose Enrique Miguens

\section{Introducción}

La historiografía de la sociología argentina ha tenido como personaje principal a Gino Germani, y como escenario privilegiado, a la Universidad de Buenos Aires (UBA). Sin embargo, frecuentemente olvidamos que este es solo un relato posible, y que el proceso de constitución de un campo relativamente autónomo de la sociología comprende también actores, espacios e instituciones que están ausentes de los principales relatos historiográficos de esta disciplina. Por esta razón, la reconstrucción de experiencias y trayectorias alternativas nos permite profundizar el conocimiento sobre el proceso de institucionalización y profesionalización de la disciplina.

Este interrogante habilita volver a trayectorias olvidadas, que configuran caminos y perfiles alternativos a los de Gino Germani. Este es el caso de José Enrique Miguens. Abogado de formación, se especializó en Sociología Económica con Parsons y Sorokin en los Estados Unidos. Docente de sociología en la Universidad de Buenos Aires (UBA) durante los primeros gobiernos peronistas, fundador de la segunda carrera de sociología del país en la Universidad Católica Argentina (UCA), e impulsor de la consultoría privada a partidos políticos y empresas, 
Miguens se revela como un actor central de la sociología argentina. Sin embargo, su historia es un capítulo aún no escrito en la historia de esta disciplina.

La realización de este trabajo se basa principalmente en cinco entrevistas que mantuvimos con José Enrique Miguens, tres en 2008, una en 2009 y una en 2010, trabajando con la metodología de historia de vida (Mallimaci y Giménez Béliveau, 2006). Al constituir la palabra del protagonista la principal fuente, siempre se corre el riesgo de adoptar el discurso de los sujetos de estudio. Es un riesgo que debemos correr, por cuanto este artículo es solo el primer paso para un estudio más profundo de la trayectoria de José Enrique Miguens. ${ }^{2}$

No es nuestra intención realizar un estudio biográfico ni una nota celebratoria de su persona. Por el contrario, su trayectoria tanto académica, intelectual, política y profesional es el material que nos permite comprender procesos más amplios, que tienen que ver con la historia de la sociología argentina y su interrelación con procesos políticos, culturales y sociales, que se hacen carne en las acciones, decisiones y representaciones de cada individuo.

En este sentido, la trayectoria de Miguens puede servir a múltiples propósitos: como perfil alternativo al de Gino Germani, como exponente de una sociología con un fuerte componente católico, como innovador de la investigación de mercado y de sondeo de opinión pública, lo cual constituye un mercado alternativo a la academia y a la función pública para los futuros sociólogos, y cuyo impacto en cómo se enseña sociología (y qué se considera sociología) en la Argentina todavía está inexplorado.

\section{Abogado en el Estado, Sociólogo en el tiempo libre}

Los comienzos de la trayectoria de José Enrique Miguens en la sociología se dan de la mano de redes sociales presentes en el espacio de los sectores tradicionales en la cual se había criado nuestro personaje. El primer Miguens nacido en la Argentina data de 1784, inscripto en la Parroquia de la Inmaculada Concepción, Buenos Aires. El padre de José Enrique Miguens era un conocido juez de la Capital Federal en la primera mitad del siglo XX, cuando el cargo de juez correspondía a un cursus honorum difícilmente accesible a individuos de sectores medios o bajos. Un hermano de José Enrique Miguens, Carlos, unió lazos con una de las principales herederas del imperio fundado por Otto Bemberg, la cineasta María Luisa Bemberg. Uno de los hijos de esta pareja, Luciano Miguens Bemberg, será presidente de la Sociedad Rural Argentina. Las redes familiares dan cuenta

\footnotetext{
${ }^{2}$ José Enrique Miguens falleció el 7 de octubre de 2011. Si bien este artículo no se propone ser un panegírico, debemos recordar la enorme generosidad de Miguens, quien accedió gustoso a los encuentros realizados en su en su departamento de la Ciudad Autónoma de Buenos Aires, mostrándose como un entrevistado amable y predispuesto, y teniendo gestos de aliento y apoyo en todo momento hacia nuestra investigación. La grandeza de Miguens quedó clara cuando, tras la ponencia de la que surge este artículo, nos hizo una serie de observaciones críticas pero sin pretender que modifiquemos nada, ya que respetaba su lugar de "actor".
} 
de los espacios en los que se debe haber socializado José Enrique, el mundo social al que pertenecía.

Nacido en Buenos Aires en 1918, José Enrique Miguens siguió la carrera de Derecho en la Universidad de Buenos Aires, al igual que su padre. Se recibe en 1943 con medalla de honor. En virtud de la amistad de su padre con Alejandro Bunge, es invitado a trabajar al grupo que el economista y estadístico mantenía en la Facultad de Ciencias Económicas de la Universidad de Buenos Aires, junto con la Revista de Economía Argentina. El llamado Grupo Bunge trascendió la muerte de su mentor en 1943, organizados en torno al Instituto Alejando E. Bunge de Investigaciones Económicas desde donde continuaron impulsando políticas nacionalistas, estatistas y principalmente industrialistas (Fernández López, 2001: 8). Allí es donde Miguens entra en contacto con la problemática a la que estará abocado las siguientes décadas: la industrialización de la argentina.

Con el golpe militar de 1943 ingresan al Estado un grupo de funcionarios con neto corte industrialista. Más allá de las disputas palaciegas, el ascenso del coronel Juan Domingo Perón conlleva la posibilidad de sumar a las filas estatales a profesionales de perfil técnico y pro industrialistas. Algunos autores afirman que este hubiera sido el momento que Alejandro Bunge esperó toda su vida (de Ímaz, 1974); pero su muerte da lugar a que aprovechen la oportunidad sus discípulos. De estas filas recluta economistas y estadísticos uno de los colaboradores más cercanos a Juan Domingo Perón, José Francisco Figuerola: este español de nacimiento era un especialista en estadísticas y legislación laboral, y fue uno de los cuadros tecnocráticos más importantes del primer peronismo. Figuerola había sido protegido de Bunge desde mediados de los años 1930. Merced a Figuerola, Emilio Llorens, Carlos García Mata, Miguel Miranda, entre otros, ingresan o impulsan su carrera en la función pública. Otros tantos lo harán en cargos de menor rango. Este es el caso de Miguens, quien comienza a ejercer como asesor jurídico en la flamante Secretaría de Industria. Su principal función era diseñar políticas aduaneras que sirvieran a la protección de la industria nacional.

En 1944, Miguens aprovecha una oportunidad que marcará su reconversión hacia la sociología. El Estado argentino tenía un incipiente sistema de becas para que algunos funcionarios realizaran estudios de postgrado en Harvard. Esta experiencia había comenzado con Raúl Prebisch en el Banco Central de la República Argentina, y se había extendido hacia la nueva Secretaría de Industria: se trataba de un programa en Public Administration, con un plan de estudios prefijado por Raúl Prebisch con fuerte orientación en política fiscal. Miguens gana la beca junto a Gabriel Meoli, compañero en la Secretaría de Industria y medalla de oro de la facultad de Ingeniería. Una vez en Harvard, se desilusiona rápidamente por la orientación matemática y economicista, y abandona el plan de estudios de Prebisch. En su lugar, participa del curso de Sociología Económica que dictaba Talcott Parsons, para rápidamente sumarse a su grupo de trabajo. En este marco, trabaja con Parsons y Pitirim Sorokin durante un año y medio en sociología económica, y colabora en la traducción al inglés del clásico de Max Weber, Economía y Sociedad, que Parsons estaba realizando (publicado en 1947). El resultado de esta experiencia en Harvard será una tesis sobre los grupos 
de intereses creados y sus oponentes en la República Argentina, un resumen del cual publicará a su vuelta en la Revista de Economía Argentina (Miguens, 1947)

No es arriesgado afirmar que es en Harvard donde Miguens a la sociología. De esta experiencia no obtendrá ningún título formal: el abandono del plan dispuesto por Prebisch anuló todo reconocimiento institucional. La única credencial que obtuvo de esta experiencia, sin embargo, es una elogiosa carta de recomendación firmada por Parsons. Este documento singular convirtió a Miguens en uno de los sociólogos argentinos con las mejores credenciales disciplinarias de la época.

De vuelta en la Argentina, retoma la carrera administrativa en la función pública en el Ministerio de Industria y Comercio, y luego en YPF. Hasta su retiro voluntario en 1958, merced a un decreto del entonces ministro de economía, Álvaro Alsogaray, Miguens cumple tareas como asesor jurídico de diversas instancias estatales. Por ejemplo, diseña proyectos de protección arancelaria, o es asesor jurídico en la Comisión Interministerial de Ministros de Obras Públicas. Es decir, más allá de que le disgustara su modus vivendi era el derecho. Mientras que a su puesto en la función pública le dedicaba gran parte de su jornada labora, la sociología quedaba relegada a una actividad de tiempo libre, ejercida en la academia.

Esta separación de actividades es típica de un contexto disciplinar de débil institucionalización y baja profesionalización. Hasta la formación de las principales carreras de sociología, y la aparición de una demanda por un saber sociológico, el ejercicio de la profesión era de carácter principalmente amateur para la gran mayoría.

Entonces, es en el ámbito universitario donde Miguens continuará su práctica como sociólogo, en las cátedras e institutos de sociología de las Facultades de Ciencias Económicas (1947-1955) y la de Derecho y Ciencias Sociales (19481953) de la Universidad de Buenos Aires (UBA).

El activismo político que profesores y estudiantes mantuvieron contra Perón en 1945 y la derrota de aquellos dio paso a una serie de represalias por parte del gobierno nacional. En 1947 cientos de profesores universitarios fueron separados de sus cargos, y reemplazados por sus adjuntos o jefes de trabajos prácticos (Blanco, 2006: 63). En este recambio, se renovarán los elencos docentes en sociología. Alberto Baldrich obtuvo en 1947 la titularidad de la cátedra de Sociología en la Facultades de Ciencias Económicas (FCE-UBA), en la que era adjunto de Ricardo Levene. Por su parte, Rodolfo Tecera del Franco es designado profesor titular de la cátedra de sociología en la Facultad de Derecho y Ciencias Sociales (FDyCS-UBA) (Blanco, 2006: 65-66; Pereyra, 2005: 28). En ambas cátedras Miguens ocupa, por concurso, el cargo de profesor adjunto. En consonancia, cuando Baldrich cree el Institutito de Sociología en la FCE-UBA en 1947, Miguens será Jefe de Investigaciones. Lo mismo ocurre en la FDyCS-UBA, donde Miguens se desempeña como coordinador junto a Tecera del Franco, Gastón Terán y Fernando Cuevillas de la Sección Sociología del Instituto de Filosofía del Derecho y Sociología (Blanco, 2006: 76)

En este punto, Miguens forma parte de un conjunto de sociólogos que dominarán la escena académica durante el decenio peronista, y que conforma una efectiva 
red institucional: en 1950 crearán la Academia Argentina de Sociología y organizán el Primera Reunión Nacional de Sociología (1950) y el Primer Congreso Latinoamericano de Sociología $(1951)^{3}$; instancias todas en las que Miguens participa activamente.

Alejandro Blanco afirma que entre ellos predominaban "las expresiones de recelo hacia la sociología empírica, y en especial, hacia la sociología norteamericana, juzgada una veces de 'practicista', otras de 'naturalista' o como 'mero catálogo de fenómenos sociales', pero en cualquier caso siempre de manera negativa." (Blanco, 2006: 64-65, 80). En términos de la práctica profesional, esta visión rechazaría a la investigación social empírica como eje central de la disciplina.

Sin embargo, Miguens presenta una nota discordante con respecto a sus pares. Producto de su experiencia en los Estados Unidos, impulsará ciertas innovaciones en el dictado de clases y en sus escritos. Por un lado, introduce bibliografía anglosajona actualizada: Parsons, Sorokin, Weber, Robert Merton y un conjunto de autores menores pero que conformaban el debate actualizado en sociología industrial en los Estados Unidos y Francia. ${ }^{4}$ Como sugiere Pereyra (2007: 5), esto pondría en cuestión la imagen de pionero que proyecta Germani, al menos en lo que refiere a la introducción de literatura sociológica anglosajona.

Junto a la utilización de bibliografía actualizada y de origen anglosajón, Miguens muestra una clara vocación de investigación empírica, que se trasluce tanto en la crítica al positivismo y empirismo vulgar, como de posiciones metafísicas que si bien serían solidarias con la sociología, constituyen materias separadas de ella. Sin embargo, Miguens no incursionará en la investigación social empírica sino hasta comienzos de la década de 1960, cuando esté en la Universidad Católica Argentina (UCA) y en el Centro de Investigaciones Motivacionales y Sociales (CIMS).

En abril de 1949 Miguens participa del Primer Congreso Nacional de Filosofía, organizado por la Universidad Nacional de Cuyo. Entre las ponencias de Hans Georg Gadamer, Rodolfo Mondolfo, Jean Hyppolite, Karl Jaspers, Benedetto Croce, Karl Löwith y Bertrand Russell entre otros, Miguens presenta un trabajo llamado "Acontecimiento y actuación en el estudio de la realidad social" en la sesión Filosofía de la Historia, la Cultura y la Sociedad, en la que encontramos a algunos de los principales sociólogos argentinos de la época. ${ }^{5}$

\footnotetext{
${ }^{3}$ En el Primer Congreso Latinoamericano de Sociología, a instancias de representantes argentinos se funda la Asociación Latinoamericana de Sociología, siendo su primer director Alfredo Poviña (Blanco, 2006); esta red regional es un escalón en los nexos que se establecen con las instancias internacionales de referencia de la disciplina: el Institut International de Sociologie (IIS) y el International Sociological Association (ISA).

${ }^{4}$ Como botón de muestra, tómese el texto Introducción a una sociología de la empresa industrial (Miguens, 1948): cita a 12 autores especializados (E. Cherrington Hughes, Roethlisberg, E. W. Burguess, George Lescure, Friedman, Schuhl, René Bolleker, John Mill, G. Canguilhem, Chester Bernard, Rogers, Van Kleek), casi todos textos de menos de dos años de publicados (entre 1945 y 1947), aparecidos en algunas de las principales revistas de sociología de la época (Cahiers Internationaux de Sociologie, American Sociological Review).

${ }^{5}$ En dicha sesión se presentan las siguientes ponencias: Alfredo Poviña "La idea sociológica de 'comunidad"'; Alberto Baldrich: "Libertad y Determinismo en el advenimiento de la sociedad política
} 
En esta ponencia Miguens comienza justificando la especificidad de la sociología frente a la metafísica: lo social tiene una realidad propia (distinta de sus manifestaciones individuales); puede ser objeto de conocimiento científico; y el sociólogo es el encargado, con conceptos propios, de estudiar sus manifestaciones (Miguens, 1949a: 1710). Luego, el autor propone dos conceptos que permiten estudiar el comportamiento humano, otorgándole unidad a la caótica realidad social: por un lado la actuación comprende "todo realizarse exterior de cualquier potencia humana", que conlleva un significado social remitido a un sujeto o unidad agente. En otras palabras, actuación es el aspecto social de todo comportamiento humano. Por otro lado, el acontecimiento refiere todo lo humano que transcurre fuera de lo individual, y que se le aparece como impuesto de los hechos. Son los datos con los que debe actuar el sujeto (Miguens, 1949a: 1711). Esta distinción permite dotar a la sociología de una vitalidad necesaria para dejar de ser una "especulación teórica de eruditos o una infructuosa búsqueda de leyes" (Miguens, 1949a: 1716). La sociología permite al hombre actuar con plena conciencia frente a los acontecimientos que se le presentan. De esta manera, la sociología contribuye a "la libertad de la vida humana personal" (Miguens, 1949a: 1716).

Esta misma mirada crítica hacia el estado de la sociología de la época en la Argentina está plasmada en El conocimiento de lo social y otros ensayos, (Miguens, 1953a), una compilación de artículos reunidos con el objetivo de "solucionar en parte al menos los problemas que tienen los estudiantes de las Universidades, y el público en general ante la falta de textos apropiados de Sociología" (Miguens, 1953a: Introducción).

Hacia fines del decenio peronista la relativa estabilidad de la que gozaba Miguens se ve alterada. Por un lado, comienza a perder terreno en la UBA. En 1953 no se le renueva el cargo de adjunto en la FDyCS-UBA. Las renovaciones eran automáticas, pero probablemente por falta de afinidad política no se renueva el cargo a Miguens. Algo similar casi sucede en FCE-UBA, pero la presión de Miguens, acompañado por los estudiantes, forzó al decano de dicha facultad a la renovación del cargo.

El conflicto del gobierno con la iglesia, factor desencadenante del golpe de Estado de 1955, fue un punto de inflexión para Miguens. Si bien nunca había votado directamente a Perón, él se consideraba Justicialista, y había apoyado al gobierno en elecciones legislativas, por coincidir con los lineamientos generales de la política del peronismo. Sin embargo, toma distancia durante la segunda presidencia, y la adhesión se quiebra cuando el enfrentamiento entre iglesia y gobierno se vuelve crítico en 1955: la identidad católica triunfa sobre la adhesión al peronismo. En uno de nuestros encuentros, Miguens lo expresó de la siguiente

argentina"; Juan Pichon-Rivière: "Distingo entre la persona y la personalidad. La persona, propiamente dicha, y sus propiedades y accidentes desde el punto de vista sociológico"; César Pico: "Los usos, causa formal de la sociedad. Sumaria exposición y justificación de la tesis de Ortega"; Julio Soler Miralles: "Ubicación de la Sociología y carácter de su enseñanza”; junto a Karl Löwith, Fortunato Brancatisano, Carneiro Leao, Harold Davis, María Delia Gatica de Motiveros y Leopoldo Eugenio Palacios. 
manera: "yo por la renta nacional no juego la vida. Pero por la iglesia sí'". A partir de allí comienza a militar activamente en contra del gobierno, en una red de católicos opositores, formada por miembros de la Acción Católica, como Ramiro de la Fuente y Florencio Arnaudo, con quienes publicará un folletín católico, Verdad, por el que el primero fue preso.

Probablemente esta militancia influyó para que, en los procesos de juicios que se iniciaron en las universidades en 1956, Miguens quedara libre de toda culpa y cargo. Sin embargo, esto no le alcanzó para conservar un puesto en la UBA. Gino Germani estaba en franco ascenso, y una de las operaciones que realiza con total éxito es apartar de su camino hacia el control de la sociología en la UBA a todo contrincante que pudiera hacerle sombra. Y ciertamente José Enrique Miguens era uno de los principales candidatos; aquella carta de Parsons era todavía una de las mejores credenciales que podía presentar cualquier sociólogo de la época en el mundo académico argentino. En el concurso por el cargo de titular de la cátedra de Sociología de la FCE-UBA, Germani logra que Miguens sea excluido por incapacidad notoria, desconocimiento de la materia y graves errores en sus libros.

La disputa entre Germani y Miguens tiene varias versiones. Miguens asegura que Germani era un poco celoso, y que confundió una ausencia de Miguens a una reunión con una señal de enemistad. Esta explicación, que conjuga el azar y lo emocional, es confrontada por la declaración de Miguel Murmis recogida por Diego Pereyra: Murmis afirma que Miguens había denunciado a Germani por comunista ante el Ministerio de Educación (Pereyra, 2005: 78), encabezado en ese momento por un católico reaccionario, Arturo Dell' Oro Maini. Por su parte, Diego Pereyra agrega que probablemente el resentimiento de Germani se remonte a 1946-1947, cuando Miguens ocupó el cargo en la FCE-UBA, al cual Germani había aplicado de la mano de Ricardo Levene (Pereyra, 2005: 69). Sea cual fuera la explicación, nos encontramos en terreno especulativo.

\section{1958-1973: Sociólogo católico y consultor privado}

Hacia fines de la década de 1950, la vida de Miguens sufre una serie de cambios. Por un lado, el retiro voluntario del Estado, encuadrado en una medida cuya intención manifiesta era reducir el déficit tanto de la administración como el de las empresas públicas (Rappoport et al, 2000). Por otro lado, la derrota en la disputa con Germani por puestos en la UBA. De esta manera, Miguens debe reconvertirse tanto académica como profesionalmente.

Paradójicamente, esta posibilidad de comenzar de nuevo consolidará su perfil como uno de los principales sociólogos de la Argentina. Esto se asienta en el carácter pionero de Miguens tanto en el ámbito académico como en lo profesional. 


\subsection{Sociólogo católico}

Por un lado, en el ámbito académico Miguens funda la segunda carrera de sociología de la Argentina en la recientemente creada Universidad Católica Argentina (UCA). Los entretelones de dicha fundación no han sido todavía explorados, a diferencia de su par en la UBA. ${ }^{6}$ Francisco Suárez, que participó como docente en esta experiencia, sugiere que se trató de una alternativa ideológica de la derecha católica frente a la izquierda liberal que se había consolidado en la UBA, hipótesis que Miguens mismo ha apoyado. De todas maneras, el proyecto de ciertos sectores del catolicismo de fundar una carrera de sociología es anterior a la fundación de la carrera por Germani (Blanco, 2006).

Más allá del motivo de fondo, Miguens es convocado por Monseñor Octavio Derisi para organizar y dirigir el Departamento de Sociología de la UCA en $1959 .{ }^{7} \mathrm{El}$ plantel de profesores, seleccionado por el propio Miguens, incluía a: Adolfo Diz, que había trabajado con Miguens en el Instituto Lanús, dictaba Estadística; Gonzalo Cárdenas se encargaba de Historia Social Argentina; Justino O'Farrel tenía a su cargo Cambio Social, Antonio Donini Introducción a la Sociología, y el propio Miguens Teoría Social Sistemática. Completaban el plantel Hugo Callelo y Francisco Suárez. Más allá de la carrera de sociología, Miguens compartiría los pasillos de la Facultad de Ciencias Sociales y Económicas con viejos compañeros del Grupo Bunge, como César H. Belaunde, Emilio Llorens, Gabriel Meoli, entre otros.

Miguens estuvo al frente del Departamento de Sociología de la UCA hasta 1966, cuando debió renunciar por un conflicto vinculado con el contexto político nacional e internacional. Cuando Onganía asume el poder, los sectores conservadores dentro de la UCA fortalecen sus posiciones y empiezan a condenar la actividad sociológica dentro de la universidad ya que no era "bien vista". En este marco, se sucede una serie de conflictos por distintos temas que terminaron desatando un éxodo masivo entre profesores y alumnos (Suárez, 1973: 120-121).

La primera tensión se desató cuando tres profesores del Departamento redactaron un manifiesto en plena guerra de Vietnam a favor de los vietnamitas. Desde el Rectorado, le pidieron a Miguens que los echara, pero éste se negó. Luego, sobrevino la prohibición del ciclo de conferencias sobre el concepto de "Cambio Social" a cargo del Lic. Hugo Callelo. El Dr. Francisco Valsecchi, decano de la Facultad de Ciencias Sociales y Económicas, ${ }^{8}$ aducía para ello que el orador poseía determinados antecedentes ideológicos que no se correspondían con el espíritu de la UCA. La última pelea se dio en torno al pedido de suspensión para el estudiante de sociología Juan José Llach (medalla de oro del curso y presidente del centro de estudiantes) por haber publicado un artículo criticando a la universidad en la revista del centro de estudiantes. Miguens se negó nuevamente

\footnotetext{
${ }^{6}$ Uno de los pocos trabajos sobre la sociología en la UCA es una ponencia presentada por Enrique Amadassi y Juan López Fidanza (2011)

${ }^{7}$ Entre los alumnos que pasaron por las aulas de Miguens podemos nombrar a Atilio Borón, Juan José Llach, Carlos Prego.

${ }^{8}$ Francisco Valsecchi fue Decano de la Facultad de Ciencias Sociales y Económicas de la UCA entre los años 1958 y 1970.
} 
a acatar el pedido de sanción aduciendo que Llach estaba próximo a recibirse. Esta fue la gota que rebalsó el vaso; Miguens decide presentar su renuncia. Cuando le comunicó a los profesores del Departamento de Sociología, la mayoría del plantel se solidarizó con él presentando también la renuncia a sus respectivos cargos. En total fueron 29 profesores en renunciar, el 95\% del cuerpo docente específico de dicho Departamento. Casi todos menos Justino O'Farrel y Francisco Suárez que mantuvieron sus cargos hasta ser echados un mes más tarde.

Además de la batalla entre Miguens y el Rectorado, podemos decir que el conflicto rebasaba el ámbito sociológico debido al malestar general producido por la intervención de las Universidades estatales por parte del Onganiato. El saldo que dejó este conflicto fue de 42 profesores de la Facultad de Ciencias Sociales y Económicas amonestados (por condenar públicamente la intervención a las Universidades estatales); 78 alumnos de Sociología, Economía y Derecho suspendidos (por idéntico motivo); dos periódicos estudiantiles fuera de circulación (Reencuentro y Sociología); carros de asalto de la Guardia de Infantería estacionados en las puertas de la UCA a pedido del Decano Valsecchi; suspensión por dos años del alumno Eduardo R. Saguier y por uno de los alumnos Roberto Martínez, Fernando Perera y Carlos Prego; éxodo voluntario de 30 de los 200 alumnos a la Universidad del Salvador, 6 a la Universidad Católica de Chile y otros a diferentes instituciones del país y el extranjero.

El conflicto en la UCA tuvo repercusión internacional. Incluso el New York Times publicó un artículo que mencionaba la renuncia de Miguens ${ }^{9}$. Luego de este revuelo, recibe una invitación de la Universidad de Notre Dame, una de las universidades católicas más prestigiosas de los Estados Unidos. Allí fue y se quedó poco menos de un año como profesor de seminarios donde se ocupaba de los siguientes temas: desarrollo económico, capitalismo, imperialismo.

Volvería a los Estados Unidos en octubre de 1971, curiosamente invitado por Germani para dar unas conferencias en la Universidad de Harvard. Se trató de unas jornadas sobre la inestabilidad de la política argentina y el mal funcionamiento de su democracia. Estas conferencias eran dirigidas a profesores y graduados de las carreras de Sociología y de Gobierno. Entre los presentes, se encontraban, además de Miguens y Germani, el economista Karl Deutsch y los politólogos Samuel Huntington y Alfred Hirschman entre quienes se armó un caluroso debate sobre los orígenes y las soluciones de la débil democracia argentina.

\subsection{Consultor privado}

Por otro lado, Miguens innova en el ámbito extraacadémico, ya que es uno de los primeros en impulsar las investigaciones de mercado y de opinión pública.

\footnotetext{
${ }^{9}$ Ver la edición del lunes 2 de enero de 1967 del New York Times, página 9: "Sociology stirs argentine furor, Professor at Catholic School Leaves Post in Protest", escrito por Barnard L. Collier.
} 
En 1958, Miguens trabaja como asesor del Instituto Lanús en la elaboración de lo que constituiría una de las primeras encuestas públicas realizadas en el país. Sin embargo, será más importante la fundación del Centro de Investigaciones Motivacionales y Sociales (CIMS), que sostiene con el año de sueldo que recibe por el retiro del Estado. EI CIMS funcionó entre 1959 y 1973, año en que Miguens vuelve a la función pública, específicamente a la Secretaría Técnica de la Presidencia de la Nación, convocado por el proyecto del peronismo.

La actividad del CIMS se prolonga por 15 años, aunque la producción se concentra fundamentalmente entre los años 1967 y 1971. Los estudios realizados y dirigidos por Miguens y su equipo, entre los que se encontraban Roberto Frenkel y Roberto Lavagna, son estudios de opinión pública y de investigación de mercado. Los temas abarcados son de un amplio espectro, por ejemplo: actitudes respecto a las privatizaciones; imágenes de partidos políticos, fuerzas armadas, Iglesia Católica; actitudes frente a las políticas petroleras; percepciones sobre los conflictos internacionales que afectaban a Argentina, con especial atención en las disputas limítrofes.

En la mayor parte de los informes también aparecen gráficos o tablas que representan los resultados de las encuestas por pregunta y por ciudad o área. La mayoría de las veces la misma encuesta es realizada en un mismo grupo de ciudades o áreas metropolitanas como por ejemplo: Capital Federal, Conurbano Bonaerense, Bahía Blanca, Córdoba, Mendoza, Resistencia, Rosario y Tucumán. Por otra parte, la mayor parte de las muestras utilizadas son estadísticamente representativas y de alcance nacional aunque también existen estudios focalizados en líderes de opinión y representantes sectoriales. EI CIMS trabaja para empresas privadas, sindicatos, la iglesia católica, partidos políticos, o el mismo Estado, nacional o provincial. Un ejemplo de esto es una investigación realizada en el marco de la intervención federal en la Provincia de Río Negro (Miguens, 1963a, 1963b).

En estos años, la producción intelectual de Miguens estará estrechamente vinculada con su labor profesional en el ámbito privado. Los temas con mayor peso son fundamentalmente el poder económico en Argentina y su vinculación con el poder político, y, por otra parte, la elaboración de una sociología que tiene como objeto central el militarismo político, entendiendo un doble proceso de politización de las fuerzas armadas y de militarización de los actores políticos.

\section{Miguens y su vinculación a redes militares}

Un acontecimiento sumamente interesante en estos años de la vida de Miguens fue su participación en 1962 como asesor en el conflicto entre fracciones de las fuerzas armadas que ha pasado a la historia como el enfrentamiento entre azules y colorados. Se trató de la confrontación de dos fracciones del Ejército Argentino para imponer la tutela sobre el gobierno constitucional de de Arturo Frondizi y delinear la política nacional. En un contexto de proscripción del peronismo, el bando Azul (comandado entonces por el Gral. Onganía) era proclive a permitir una 
limitada actividad política de algunos dirigentes justicialistas, con el doble fin de lograr la normalización institucional y detener la radicalización de los grupos de "extrema izquierda". Por su parte, los Colorados abogaban por aniquilar completamente al movimiento peronista. Este conflicto, que tendrá coletazos en 1963, se cierra con el triunfo del Bando Azul, lo que significa un recambio generacional en la conducción de las Fuerzas Armadas y el desplazamiento de los sectores más recalcitrantes frente al peronismo (Portantiero, 1977). En este marco, Miguens se unirá al bando legalista, los azules.

En el cuartel de Campo de Mayo, Miguens se presenta como sociólogo y profesor, por lo que es sumado a la Sección de Acción Psicológica, dirigida por el coronel José María Díaz. Esta sección ocupa dos estaciones de radio de la Capital Federal con el objetivo de difundir 149 comunicados por radio cada media hora (Springer, 1968). Miguens es el encargado, merced a su expertise, de redactar dichos comunicados y la consigna: "estamos dispuestos a luchar para que el pueblo pueda votar. ¿Está Ud. dispuesto a luchar para que no vote?". Miguens adjudica a la claridad de dicha consigna gran parte de la victoria del bando legalista. A partir de esta experiencia, Miguens se convertirá en asesor del ministro del Interior en las reformas tomadas en contra de los amotinados.

Esta intervención de Miguens en conflictos y espacios militares debe enmarcarse no en una espontánea iniciativa del sociólogo asesor, sino en la del sociólogo comprometido con proyectos políticos en los que las fuerzas armadas eran el actor central. En concreto, Miguens participará de espacios vinculados con la fracción del Ejército Argentino que, liderado por Onganía, tomará el poder en junio de 1966.

La participación de Miguens en espacios de sociabilidad y redes militares comienza a fines de la década de 1950: desde 1958 era profesor de Sociología en la Escuela Superior de Guerra (ESG). Es altamente probable que allí haya entrado en contacto con otros profesores y militares activados políticamente, como Mariano Grondona, quien adhiere activamente por el bando Azul y se convierte luego en vocero de la Revolución Argentina. De la misma manera, estas redes con altos componentes militares probablemente hayan sido decisivas para que durante el gobierno de Onganía, Miguens participara, en el marco del Consejo Nacional de Seguridad (CONASE), en la producción del documento Lineamientos de un nuevo proyecto nacional bajo la coordinación del coronel Oscar Grondona (Castelucci, 2005: 92-93) ${ }^{10}$

El mismo tipo de redes atraviesa la participación de Miguens en la Instituto para el Desarrollo Empresarial en la Argentina (IDEA). Merced a un artículo publicado en la revista Metalurgia (Miguens, 1965b), Miguens se incorpora como coordinador de los cursos, donde logra crear una escuela de administración privada, para luego

\footnotetext{
${ }^{10}$ Lineamientos de un nuevo proyecto nacional es el principal documento publicado por un grupo de especialistas compuesto por: Javier Villanueva, Horacio Pietranera, Juan Carlos Puig, Julio Grondona y José Luis Machinea, Carlos Floria, José Enrique Miguens, Emilio Fermín, Emilio Mignone, Mario Brodersohn, Eduardo Tiscornia, Venancio Carullo, Tulio Loza, Natalio Botana, Adolfo Critto y Héctor Ciapuscio, J. L. Dios y H. Greenun. El equipo estaba coordinado por el coronel Oscar Grondona.
} 
acceder a la presidencia de IDEA. Esto significa que Miguens preside una de las principales asociaciones de empresarios de la Argentina entre 1968 y 1973, en cuyos seminarios y reuniones circulaban políticos y empresarios ligados al gobierno militar

Miguens dejará IDEA cuando vuelva al Estado de la mano de miembros de estas mismas redes militares. Miguens había entrado en contacto con Vicente Damasco, quien había cursado estudios en la ESG, y que en el último lustro de la década de 1960 tuvo a su cargo la tarea de establecer vínculos con los profesores y conferencistas que daban clase en esa institución. Estas interacciones entre civiles y militares tenderán redes de las cuales saldrán varios cuadros que nutrirán al tercer gobierno de Juan Domingo Perón; el nexo lo hace Damasco, que se convierte en cercano colaborador del líder Justicialista. En febrero de 1974, Damasco es nombrado al frente de la Secretaría de Gobierno, y convoca a un grupo de asesores para que lo acompañen en la redacción del Modelo Argentino para el Proyecto Nacional, considerado el último documento de Perón (Castelucci, 2005) Entre los asesores se encuentra José Enrique Miguens, quien permanecerá poco menos de un año en ese puesto: en parte desalentado por la situación política del país, hacia fines de 1974 viaja a los Estados Unidos invitado por la Universidad de Connecticut, donde permanecerá un año dando clases. A su regreso se jubilará.

\section{La dimensión católica de la trayectoria de Miguens}

A lo largo de su vida, Miguens participa de diversos espacios y redes que contienen fuertemente elementos católicos.

En un primer momento, el Grupo Bunge constituye un primer espacio claramente católico: además del mismo Bunge, varios de los miembros más importantes de dicho grupo eran militantes católicos, por ejemplo José Francisco Figuerola, Emilio Llorens, Correa Ávila. El ejemplo más claro de esto está en el libro Soluciones Argentinas a los problemas económicos y sociales del presente (AAVV, 1945), el único documento publicado por el Instituto Alejando E. Bunge de Investigaciones Económicas y Sociales. En este libro, el componente católico sirve de sustrato para pensar diversos problemas de la sociedad argentina, así como sus soluciones. Miguens es coautor de dos de los artículos. En uno de ellos, por ejemplo, ante el problema del aumento de la natalidad extramatrimonial los autores proponen enviar a sacerdotes a misionar por todo el país, otorgándoles funciones y poderes de funcionarios públicos del Registro Civil (para así consagrar en matrimonio a todas las parejas). Asimismo, como hemos mencionado el Grupo Bunge servirá de plataforma de ingreso hacia el Estado de sus miembros.

Durante la década de 1940 el nombre de Miguens está asociado a varias iniciativas en el campo cultural e intelectual provenientes del catolicismo. Entre 1942 y 1950 forma parte del Consejo Superior de la Acción Católica Argentina. En 1949 forma, junto a Juan Pichón-Rivière, la Asociación Católica de Filosofía. Finalmente, en 1951 está mencionado en el proyecto de formación de una Escuela 
de Ciencias Sociales en el marco del Instituto de Cultura Católica (Blanco, 2005: 66), nombre con el que habían sido rebautizados los Cursos de Cultura Católica.

Las situaciones límites suelen forzar a los individuos a optar: el conflicto entre el Gobierno Peronista y la Iglesia Católica en 1954-1955 pondrá de manifiesto la identificación católica de Miguens. Al igual que numerosos católicos que habían tenido cierta afinidad con el peronismo, Miguens vive este enfrentamiento como un punto de quiebre, en el que es forzoso elegir bando. Por ello, militará activamente en contra del gobierno peronista desde el campo cultural, editando un folletín político. Sin embargo, veremos que esta ruptura no es definitiva: Miguens volverá al Peronismo en la 1973, y será la primera (y única) vez en la que votará a Perón como presidente de la República.

Finalmente, el acontecimiento que más fuerte marca la cercanía de Miguens a los diversos proyectos del catolicismo es la invitación por parte de Monseñor Octavio Derisi, en 1958, para formar parte del proyecto fundador de la Universidad Católica Argentina. En particular, le será encargada la creación del Departamento de Sociología y de la Carrera de Sociología. Este no es un hecho menor: se trata de la segunda carrera de sociología en ser creada tras la de la Universidad de Buenos Aires.

Además de participar en espacios profesionales y académicos de corte católico, Miguens publica en varias revistas abiertamente católicas: en 1948 y 1949 publica sendos artículos en la revista Ciencia y $\mathrm{Fe}^{11}$. Posteriormente, publicará en Criterio, y en la revista de la institución jesuita Centro de Investigación y Acción Social, y varios de sus libros saldrán bajo editoriales católicas como Ciudad y Espíritu, y Plus Ultra.

Todo esto nos lleva a considerar a José Enrique Miguens como un cuadro intelectual católico. Se trata de individuos socializados política y culturalmente en diversos espacios del catolicismo (Donatello, 2011), que participarán activamente de la vida pública pero no de instituciones propiamente religiosas. Su visión del mundo está fuertemente arraigada en un imaginario católico. El caso de Miguens responde a un patrón de época: militantes católicos que ocupan cargos en la burocracia estatal, y que se ven seducidos por el proyecto de país peronista (Donatello, 2005: 104; Cucchetti, 2005: 16), para quienes "su ser religioso significa presencia social y política en movimientos que no atenten contra sus creencia e identidad católica. [En este sentido,] (...) no se incorporan a experiencias 'cristianas' sino que se suman a aquellas de 'inspiración cristiana'" (Mallimaci, 2001: 225). De hecho, se puede delinea una trayectoria colectiva (aunque no grupal): militantes juveniles en dispositivos del catolicismo integral (Acción Católica, Cursos de Cultura Católica, etc.); titulados en Derecho en universidades estatales; luego orientados hacia las ciencias sociales (sociología, ciencia política). Antiperonistas en 1955, y afines al bando Azul en 1962. Algunos de ellos se insertan en redes militares, sea por familiares en servicio, o porque, entre fines de

\footnotetext{
${ }^{11}$ La revista Ciencia y Fe fue fundada en 1944 como continuación de la revista Strómata. Editada y dirigida por la Compañía de Jesús de San Miguel, provincia de Buenos Aires, se trata de una publicación avocada a temas filosóficos que circulaba por diversas facultades.
} 
los 50's y comienzos de los 60's dictan clases en institutos militares (Escuela Superior de Guerra, etc.). Al mismo tiempo, se desempeñan como docentes en las universidades católicas (UCA, USAL). En 1966 acompañarán al golpe de Estado, y muchos de ellos ocuparán diversos cargos en dicho gobierno. Hacia mediados de 1970, ninguno de ellos permanecía en el gobierno. Con matices, este es el caso de Raúl Puigbó, Mariano Grondona, Julio E. Álvarez, José Enrique Miguens, Guillermo Borda, Carlos Floria, José Luis de Ímaz, Emilio Mignone, José Manuel Saravia (h), entre otros (Giorgi, 2010). Estos nombres forman parte de diversos grupos católicos y provienen de tradiciones político-partidarias múltiples. Esta trayectoria colectiva de ninguna manera debe suponer una comunidad de ideales, un mismo horizonte de sentido; sin embargo, compartían una difusa adhesión a cierto imaginario católico. Más importante, esto es un claro ejemplo de una red social sustentada sobre vínculos débiles (Granovetter, 1973).

\section{A modo de cierre}

Este artículo constituye una primera reconstrucción de la trayectoria de José Enrique Miguens. En estas páginas pretendemos no más que comenzar a asentar una línea de estudio. Por ello, hemos trabajo los principales hechos que han surgido de las entrevistas que mantuvimos con Miguens, aquellos que han llamado más nuestra atención. De ningún modo pretendemos ser exhaustivos: hay numerosos puntos sobre los cuales profundizar, y a esto nos avocaremos en próximos trabajos.

Sin embargo, podemos realizar algunos comentarios que resultan ineludibles en esta trayectoria. En sus primeros años, Miguens es un converso: un abogado que se forma en ciencias económicas pero con clara orientación sociológica. El resultado será un asesor jurídico en cuestiones industrialistas, que da clases de sociología en dos facultades con magra producción escrita. Esto se debe, a riesgo de señalar lo obvio, a que la disponibilidad de tiempo para estudiar, leer, investigar, debatir y reflexionar es mínima frente a las exigencias de un trabajo que poco y nada tiene que ver con la producción de docencia e investigación académica de un sociólogo de la época. Recién cuando pueda vivir de y para la sociología, es decir cuando crea el CIMS, la producción escrita de Miguens crecerá.

En este sentido, las preocupaciones de la obra de Miguens van acompañando los cambios que sufre la Argentina a lo largo de la segunda mitad del Siglo XX. En las décadas de 1940 y 1950 sus escritos giraban en torno a las consecuencias sociales de la industrialización, en el campo de la sociología económica. Luego, en los años 1960 y 1970 se inclinará por el estudio de grupos de poder, especialmente el rol político de las fuerzas armadas, integrando la sociología política, la sociología económica y la sociología sobre las fuerzas armadas. Finalmente, en las décadas de 1980 y 1990 el grueso de su literatura tratará la salida de la dictadura (sin olvidar el papel de los militares) y la democracia como sistema. Tanto en el primer como en el segundo bloque temático podemos ver paralelismos entre los temas y la actividad profesional en la que se desempeñaba 
Miguens: primer como funcionario asesor jurídico en el área de industria del Estado Nacional, y luego como consultor y encuestador privador en el CIMS, los cuales responden en gran parte a los intereses y pedidos de aquellos que los encargaban. De manera análoga, la carrera de sociología de la UCA, que él dirigirá, tendrá un fuerte énfasis en la formación metodológica.

Por otra parte, si hacemos hincapié en la dimensión religiosa de la trayectoria de José Enrique Miguens es porque este elemento es central para comprender tanto las redes en las que circula, los espacios en los que se inserta y su producción intelectual. Su perfil se encuentra alejado de los católicos liberales, que relegan lo religioso al mundo de lo privado, pero también de los católicos integrales que hacen de la religión un componente fundamental en la construcción identitaria. Ubicado entre estos dos extremos, la dimensión católica en trayectoria de José Enrique Miguens está presente en las redes en las que circula, y permea parte de su producción intelectual.

Sin lugar a dudas José Enrique Miguens es un personaje complejo. A lo largo de 30 años pudimos ver cómo Miguens dicta clases en la universidad pública laica, en la universidad privada confesional, en espacios militares y en espacios empresariales; es formador de abogados, economistas, sociólogos, empresarios y militares. Con fundamento puede ser tenido como par de Gino Germani en la institucionalización de la sociología en la Argentina, así como pionero de la investigación de mercado y de las encuestas de opinión pública. Como funcionario del Estado fue tanto burócrata como asesor político del príncipe. Su compromiso político zigzaguea: es funcionario del primer gobierno peronista, conspira activamente para derrocar al segundo gobierno peronista, y se suma entusiasmado al tercero. Apuesta a éste proyecto; así como también había actuado en un cargo técnico durante la Revolución Argentina. Militante católico, adherente intermitente al peronismo, el pensamiento de Miguens tiene elementos corporativistas, desarrollistas, en el que la Doctrina Social de la Iglesia se trasluce en el rechazo por el liberalismo individualista. Quizá esta heterogeneidad responda al hecho de haber participado en la ecléctica política Argentina de la última mitad del siglo XX desde una posición híbrida entre el intelectual académico y el consultor experto. En este punto, las aparentes contradicciones que podamos encontrar en la trayectoria de Miguens no son más que las de un sujeto atravesado por algunas de las múltiples contradicciones de la historia argentina del siglo XX. 


\section{Referencias bibliográficas}

(2011) AMADASI, Enrique y LÓPEZ FIDANZA, Juan M., "La UCA y la sociología en la UCA, desde sus inicios hasta nuestros días", ponencia presentada en las VIII Jornadas de Sociología de la UBA, noviembre.

(1945) AAVV, Soluciones Argentinas a los problemas económicos y sociales del presente, Instituto Alejando E. Bunge de Investigaciones Económicas y Sociales, Ed. Kraf, Buenos Aires.

(2006) BLANCO, Alejandro, Razón y Modernidad, Siglo XXI, Buenos Aires.

(2005) CASTELUCCI, Oscar, “¿Cómo y por qué Juan Domingo Perón escribió Modelo argentino para el proyecto nacional?". En Juan Perón modelo argentino para el proyecto nacional, Sudamericana y COPPPAL-Argentina, Buenos Aires

(2005) CUCCHETTI, Humberto, Religión y política en Argentina y en Mendoza (1943 - 1955): lo religioso en el primer peronismo, Ceil-Piette-CONICET, Buenos Aires

(1974) DE IMAZ, José Luis, "Alejandro E. Bunge, economista y sociólogo (18801943)", Desarrollo Económico, Vol. 14, № 55, octubre-diciembre, pp. 545-567.

(2005) DONATELLO, Luis, El Catolicismo Liberacionista en la Argentina y sus opciones político-religiosas, tesis doctoral, Facultad de Ciencias Sociales, Universidad de Buenos Aires y Écoles des Hautes Études en Sciences Sociales, Francia, mimeo.

(2011) DONATELLO, Luis Miguel, "Del antiliberalismo al neoliberalismo. Del nacionalismo al comunitarismo: los 'cuadros' católicos en la Argentina del siglo XX", en: PÉREZ, G., AELO, O. y SALERNO, G. (comp.), Todo aquel fulgor, Editorial Nueva Trilce, Buenos Aires.

(2001) FERNÁNDEZ LÓPEZ, Manuel, "La ciencia económica argentina en el siglo XX", Estudios Económicos, Vol. XVIII, n³ 38, julio-diciembre, pp. 1-30.

(2010) GIORGI, Guido, "Redes católicas y Estado en la 'Revolución Argentina”, Ciencias Sociales y Religión, Vol. 12, № 12, pp. 53-78.

(1973) GRANOVETTER, Mark, "The strength of weak ties", American Journal of Sociology, Volume 78, №6, Chicago, pp. 1360-1380

(2001) MALLIMACI, Fortunato, "Los diversos catolicismos en los orígenes de la experiencia peronista". En MALLIMACI, Fortunato y DI STEFANO, Roberto (comp.), Religión e imaginario social, Manantial, Buenos Aires.

(2006) MALLIMACI, Fortunato y GIMÉNEZ BÉLIVEAU, Verónica, "Historias de vida y método biográfico". En Vasilachis de Gialdino, Irene (coord.), Estrategias de investigación cualitativa, Gedisa, Buenos Aires, pp.179-209. 
(1948) MIGUENS, José Enrique Introducción a una sociología de la empresa industrial, Universidad de Buenos Aires. Cuadernos del Instituto de Sociología, Buenos Aires.

(1949) MIGUENS, José Enrique, "Acontecimiento y actuación en el estudio de la realidad social", Universidad Nacional de Cuyo, Separata de las Actas del $1^{\circ}$ Congreso Nacional de Filosofía, Mendoza.

(1953) MIGUENS, José Enrique, El conocimiento de lo social y otros ensayos, Perrot, Buenos Aires.

(1963a) MIGUENS, José Enrique, Enumeración y valorización de las instituciones y servicios por departamentos. Intervención federal en la provincia de Río Negro., Secretaría Técnica, Ministerio de Asuntos Sociales, Viedma.

(1963b) MIGUENS, José Enrique, Posibles desplazamientos demográficos en la Provincia de Río Negro, Secretaría Técnica, Ministerio de Asuntos Sociales, Viedma.

(2005) PEREYRA, Diego International Networks and the Institutionalisation of Sociology in Argentina (1940-1963), Tesis doctoral, School of Social Sciences and Cultural Studies, University of Sussex at Brighton, mimeo.

(2007) PEREYRA, Diego, "Cincuenta Años de la Carrera de Sociología de la UBA. Algunas notas contra-celebratorias para repensar la historia de la sociología en Argentina", mimeo.

(1977) PORTANTIERO, Juan Carlos "Economía y política en la crisis argentina, 1958-1973", Revista Mexicana de Sociología, Volumen 39, № 2, México D.F., pp. 301-340.

(2000) RAPOPORT, Mario. et al, Historia económica, social y política de la Argentina (1880- 2000), Macchi, Buenos Aires.

(1968) SPRINGER, Philip B., "Disunity and Disorder: Factional Politics in the Argentine Military". En Bienen, Henry (ed.), The military intervenes: Case studies in political development, Russell Sage Foundation, New York.

(1973) SUÁREZ, Francisco, "Algunas reflexiones sobre los procesos de institucionalización de la Sociología en la Argentina durante los últimos años", Revista Mexicana de Sociología, Vol. 35, No 1, enero-marzo, México D.F., pp. 117134. 